two time periods. $56 \%$ reduced readmission rates for Ischemic Stroke performance and $47 \%$ reduced readmission rates for Hemorrhagic Stroke performance.

Notes (figure 1): Analysis excludes $0-17$ age group and includes the neurosciences and spine service lines and the Brain/Central Nervous System (CNS) Cancer CARE Family from the cancer service line. CNS injury includes concussion, late effects of neuro trauma, paralysis, skull fracture and major brain injury, and spinal cord injury. Movement disorders include Parkinson disease, movement disorders, multiple sclerosis and demyelinating diseases. Neuro pain disorders include headache/migraine, neuro pain and neuropathy. Other includes hydrocephalus and spina bifida, neurologic disease-other, and sleep disorders. Stroke and neurovascular include ischemic and hemorrhagic stroke, transient ischemic attack, and neurovascular diseases. Sources: Impact of Change ${ }^{\circledR}$, 2018; HCUP National Inpatient Sample (NIS). Healthcare Cost and Utilization Project (HCUP) 2015. Agency for Healthcare Research and Quality, Rockville, MD; Claritas PopFacts $^{\circledR}$, 2018; Circulation. 2019;139:e56-e528. DOI: 10.1161/CIR.0000000000000659; Sg2 Analysis, 2018.

Notes (figure 2): $n=32$. Ischemic Stroke ICD 10 Codes. Hemorrhagic Stroke ICD 10 codes. Evaluated 30 day unplanned, all cause all payer readmission rates (adults, 18 years and older). Comparisons amongst facilities is not advised since a select number of facilities met the exclusion criteria. Exclusion criteria: total discharges for at least one period was equal or less than 20 discharges. Considerations for interpreting results: volume fluctuations did occur by hospital type (primary stroke centers vs. comprehensive stroke centers), which may impact the $\%$ difference when calculating readmissions. Source: Vizient Clinical Data Base/Resource Manager ${ }^{\mathrm{TM}}$

Notes (figure 3): $N=19$. Ischemic Stroke ICD 10 Codes. Hemorrhagic Stroke ICD 10 codes. Evaluated 30 day unplanned, all cause all payer readmission rates (adults, 18 years and older). Comparisons amongst facilities is not advised since a select number of facilities met the exclusion criteria. Exclusion criteria: total discharges for at least one period was equal or less than 10 discharges. Considerations for interpreting results: volume fluctuations did occur by hospital type (primary stroke centers vs. comprehensive stroke centers), which may impact the $\%$ difference when calculating readmissions. Source: Vizient Clinical Data Base/Resource Manager ${ }^{\mathrm{TM}}$
Conclusions Key learnings for success were prioritized to reduce clinical variations in care for stroke patients and optimize system-wide transitions in care (figure 4). This collaborative model effectively identified best practices to reduce variations in stroke care while preparing for emerging advancements in technology, guidelines and new care delivery payment models.

\section{UTILIZING LEAN SIX SIGMA AND QUALITY IMPROVEMENT (QI) TO INCREASE DIABETES SELF- MANAGEMENT EDUCATION REFERRALS WITHIN THE ENDOCRINOLOGY CLINIC}

${ }^{1}$ Jessica Burks, ${ }^{1}$ Usha Kollipara, ${ }^{1}$ Jacqueline Mutz, ${ }^{1} J a s o n$ Fish, ${ }^{2}$ Estefania Andrioaia Lopez, ${ }^{2}$ Jessica Abramowitz, ${ }^{2}$ Nancy Drobycki, ${ }^{2}$ Sadia Ali. ' Southwestern Health Resources, USA; ${ }^{2}$ UT Southwestern Medical Center, USA

\subsection{6/bmjoq-2020-IHI.18}

Background Diabetes self-management education (DSME) is the ongoing process of facilitating the knowledge, skill, and ability necessary for diabetes self-care. Despite evidence that DSME improves clinical outcomes, health status, and quality of life for persons with diabetes referrals to DSME continue to be low in our population.

Objectives To increase referrals to Diabetes Self-Management Education (DSME) program from 26\% at baseline to at least $40 \%$ in 12 months in our Endocrinology Clinic.

Methods We utilized Six Sigma DMAIC (Define-Measure-Analyze-Improve-Control) methodology to problem solve and perform process improvement. We created a multi-functional team including clinic staff, providers and quality improvement personnel to assist in the design and development of the new processes. In define phase we generated a project charter and communication plan. During the measure phase we determined the baseline referral volume, completed a current state process map and completed measurement system analysis. During the analyze phase we brainstormed reasons for low referrals (root cause analysis) and analyzed referral volume for trends. During improve phase we finalized future state workflow, implemented pre-visit planning, standing medical orders for referrals and provided training to clinic staff and 
providers. We continued bi-weekly chart audits for appropriate referrals being placed for 12 months.

Results As of August 2020, we were able to meet our goal at $42 \%$ with 1,813 referrals placed for DSME. Of these, 1,086 patients completed their education sessions (figure 1).

Conclusions Quality improvement tools and methodologies can be successfully utilized to improve care for diabetes in clinical settings. We were able to create a process that has maintained success, even during the uncertain times of COVID-19. The processes created and put in place were sustainable and have allowed the staff to continue placing referrals during telehealth visits and patients completing DSME virtually.

\section{DECREASING NEONATAL JAUNDICE READMISSION RATES THROUGH IMPLEMENTATION OF A JAUNDICE MANAGEMENT GUIDE}

${ }^{1}$ Laura Hunt, ${ }^{1}$ Marie Ramos, ${ }^{1} Y$ vonne Helland, ${ }^{2}$ Karen Lamkin. ${ }^{1}$ Baylor Scott and White Health, USA; ${ }^{2}$ Baylor Scott and White at Waxahachie, USA

\subsection{6/bmjoq-2020-|HI.19}

Background Baylor Scott and White Medical Center at Waxahachie (BSWMCW), a level 1 neonatal designated facility, had rising neonatal jaundice readmission rates for two fiscal years $(1.7 \%-2.6 \%)$. Based off literature review, this was above the average rate for hospitals with similar populations $(0.5 \%-2.0 \%)$. Neonatal jaundice readmissions have several effects on families and the neonate related to increased costs, stress on bonding, and increased risk for hospital acquired infections.
Objectives By June 30, 2019, BSWMCW will reduce the rolling neonatal jaundice readmission rate from $2.6 \%$ (July 1, 2017-June 30, 2018) to less than 1\% (July 1, 2018 - June 30, 2019).

Methods A multidisciplinary committee met to identify why excessive neonatal jaundice readmissions occurred. Issues identified were inconsistent practices in starting and stopping phototherapy, timing of discharge, and timing of newborn follow up in infants with jaundice (figure 1). The team prioritized management of jaundice through recognizing plan of care modifications during the initial hospitalization which would impact newborn readmission rates. Initially, clear guidelines were created on when to start or stop phototherapy, including discharge criteria, followed by implementation of new supplementation guidelines. Donor milk was offered as an alternative to formula supplementation. In order to adhere to supplementation guidelines, standardized newborn weights at 24 hours of life was added. Finally, jaundice lab testing was required through BSWMCW inpatient lab prior to admission rather

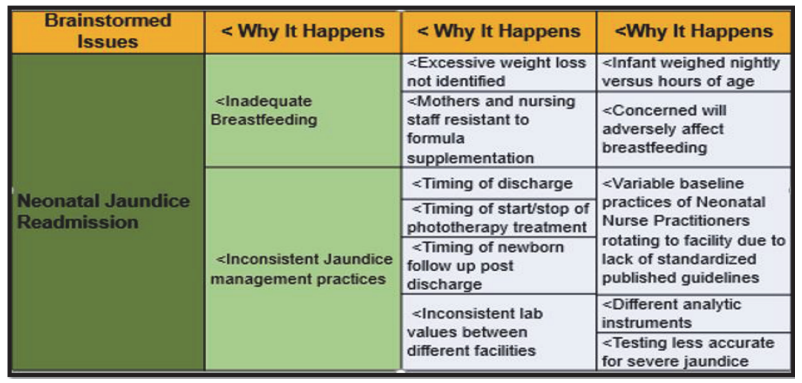

Abstract 19 Figure 1

Decreasing Neonatal Jaundice Readmission Rates Through Implementation Of A Jaundice Management Guide

\begin{tabular}{|c|c|}
\hline & JAUNDICE MANAGEMENT GUIDE \\
\hline \multicolumn{2}{|r|}{ Indications to Start Phototherapyfor Inpatients } \\
\hline \multicolumn{2}{|c|}{ 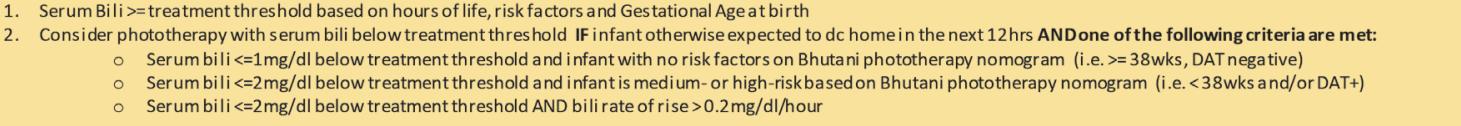 } \\
\hline \multicolumn{2}{|r|}{ How to Provide Phototherapy } \\
\hline Bilirubin Level & Treatment \\
\hline Bel ow treatment threshold & ○ Biliblanket \\
\hline At threshold & - bili blanket +/- overhead light, depending on rate of rise, risk factors, target day of dc, etc. \\
\hline Above treatment threshold & $\circ$ Biliblanket and at least one overhead light \\
\hline Bili within $3-4 \mathrm{mg} / \mathrm{dl}$ of threshold for exchange transfusion & $\circ$ Biliblanket and at least two overhead lights \\
\hline Bili $>=3$ of treatment & $\begin{array}{l}3 \text { total lights needed ((i.e. blanket and double ba nk overhead) and/or infant needing IVF and/or any } \\
\text { concerns for poor parental compliance with trea tment: trea tment should occur physically in intermediate } \\
\text { carenursery) }\end{array}$ \\
\hline \multicolumn{2}{|c|}{$\begin{array}{l}\text { *Outpati ent phototherapy can be considered ONLY if no riskfactors a re pres ent (i.e. full term, no ABO set-up, etc.), feeding is going well a nd fa mily is reliable with gooda ccess to } \\
\text { care }\end{array}$} \\
\hline \multicolumn{2}{|r|}{ Stopping Phototherapy } \\
\hline Estimated Gestational Age $>=38$ weeks at birth & - Serum bili $>=3 \mathrm{mg} / \mathrm{dl}$ bel ow treatment threshold for current hours of life \\
\hline Estimated Gestational Age $<38$ weeks at birth & - Serum bili $>=4 \mathrm{mg} / \mathrm{dl}$ bel ow treatment threshold for current hours of life \\
\hline \multicolumn{2}{|c|}{$\begin{array}{l}\text { Schedule follow-up within } 24 \mathrm{hrs} \text { if bili is high intermediate risk and continuing to rise and/or if infant has persistent feeding difficulties (i.e. excessive weight loss, not latching } \\
\text { well, etc.) associated with jaundice; otherwise follow-up should be scheduled within } 48 \text { hours. } \\
\text { Determining Readiness for Discharge }\end{array}$} \\
\hline \multirow{2}{*}{\multicolumn{2}{|c|}{ 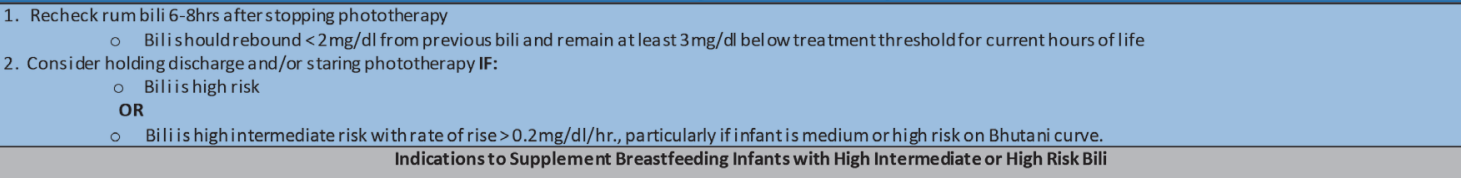 }} \\
\hline & \\
\hline 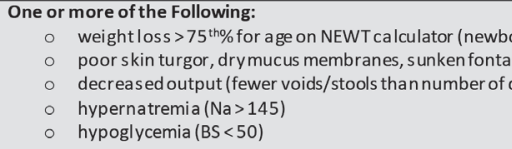 & $\begin{array}{l}\text { ght.org) and } / o r>=7^{\text {tho }} \text { at } 24 \mathrm{~h} \\
\text { rother clinical si } / \mathrm{sx} \text { of deh ydration } \\
\text { d) }\end{array}$ \\
\hline
\end{tabular}

